Bundesgesundheitsbl 2017 · 60:737-744 DOI 10.1007/s00103-017-2564-3

Online publiziert: 18. Mai 2017

(c) Der/die Autor(en) 2017. Dieser Artikel ist eine Open-Access-Publikation.

CrossMark

Susanne Andres - Katharina Schultrich • Bernhard Monien - Thorsten Buhrke • Nadiya Bakhiya · Falko Frenzel · Klaus Abraham · Bernd Schäfer · Alfonso Lampen Bundesinstitut für Risikobewertung, Berlin, Deutschland

\title{
Erhitzungsbedingte Kontaminanten in Lebensmitteln
}

\section{Acrylamid, Furan und Fettsäureester von Monochlorpropandiolen und Glycidol}

Vorkommens im Lebensmittel sowie zur Toxikologie bekannt ist [3].

Der Fokus dieses Beitrags liegt auf den bisher am besten untersuchten erhitzungsbedingten Kontaminanten Acrylamid, Furan und den Fettsäureestern von Glycidol sowie Monochlorpropandiolen (MCPD), zu denen das 2-MCPD und das 3-MCPD zählen. Die Mehrzahl der genannten Verbindungen erwies sich in tierexperimentellen Untersuchungen bei Langzeitapplikation von hohen Dosierungen als kanzerogen. Die - Tab. 1 zeigt eine Übersicht zur Entstehung der Verbindungen, zu den hauptsächlich betroffenen Lebensmitteln, zu den Effekten im Tierexperiment und zur Regulation.

Weiterhin werden Bewertungskonzepte diskutiert, die zu einer schrittweisen Priorisierung des Gefährdungspotenzials für die Vielzahl an erhitzungsbedingten Kontaminanten in Lebensmitteln führen sollen. Für die meisten identifizierten Verbindungen ist bezüglich ihres Vorkommens im Lebensmittel sowie zu ihrer Toxikologie nur sehr wenig bekannt, sodass eine umfassende Risikobewertung für jede einzelne Verbindung nur langfristig realisierbar ist.

\section{Acrylamid}

Acrylamid (•Abb. 1) wurde erstmals 2002 von einer schwedischen Arbeitsgruppe in einer Vielzahl von Lebensmitteln nachgewiesen. Es entsteht unter anderem bei der Zubereitung bzw. Verarbeitung stärkehaltiger Lebensmittel bei hohen Temperaturen von über $120^{\circ} \mathrm{C}$ (Braten, Backen, Frittieren oder Rösten) und geringer Feuchtigkeit bei der Reaktion von reduzierenden Zuckern (z. B. Glukose oder Fruktose) und bestimmten in Lebensmitteln natürlich vorkommenden Aminosäuren (vor allem Asparagin). Besonders hohe Gehalte an Acrylamid finden sich in Kartoffelprodukten wie Pommes frites und Kartoffelchips, in Cerealien, in Brot (vor allem Knäckebrot) sowie in Kaffee. Eine weitere wesentliche Expositionsquelle stellt die inhalative Exposition über Zigarettenrauch dar (auch durch Passivrauchen; [4-6]).

In Säugetieren wird die Substanz hauptsächlich durch Cytochrom P4502E1 (CYP2E1) zu Glycidamid umgesetzt, das ein deutlich höheres gentoxisches Potenzial als die Ausgangssubstanz aufweist. Hauptstoffwechselweg von Acrylamid und Glycidamid beim Menschen ist die Konjugation mit Glutathion. Die letztlich entstehenden Merkaptursäuren sind als Kurzzeitbiomarker im Urin nachweisbar $[7,8]$.

Toxikologisch besonders relevant ist die Fähigkeit von Acrylamid und vor allem des gebildeten Glycidamids, mit Thiol- sowie Aminogruppen (z. B. Glutathion, Hämoglobin) und mit Basen der Desoxyribonukleinsäure (DNA) zu reagieren. In der modernen Toxikologie werden Acrylamid- und GlycidamidHämoglobin-Addukte in den roten Blutkörperchen als Biomarker für die mittelfristige individuelle Exposition herangezogen. In bakteriellen Testsystemen und verschiedenen zellkulturbasierten Testsystemen erwies sich Acrylamid 
Leitthema

Tab. 1 Übersicht zu Entstehung, Vorkommen, Toxikologie und Regulierungsprinzipien von erhitzungsbedingten Kontaminanten in Lebensmitteln

\begin{tabular}{|c|c|c|c|c|c|}
\hline Kontaminante & Acrylamid & Furan & $\begin{array}{l}\text { Glycidyl- } \\
\text { Fettsäureester }\end{array}$ & $\begin{array}{l}\text { 3-MCPD/3-MCPD- } \\
\text { Fettsäureester }\end{array}$ & $\begin{array}{l}\text { 2-MCPD/2-MCPD- } \\
\text { Fettsäureester }\end{array}$ \\
\hline Entstehung & $\begin{array}{l}\text { Braten, Backen, Rös- } \\
\text { ten, Frittieren von } \\
\text { stärkehaltigen Le- } \\
\text { bensmitteln }\end{array}$ & $\begin{array}{l}\text { Bräunungsvorgänge } \\
\text { (z. B. Maillard-Reakti- } \\
\text { on) }\end{array}$ & $\begin{array}{l}\text { Raffination von Speise- } \\
\text { fetten/-ölen }\end{array}$ & $\begin{array}{l}\text { Raffination von Spei- } \\
\text { sefetten/-ölen; Her- } \\
\text { stellung hydrolysierter } \\
\text { Pflanzenproteine }\end{array}$ & $\begin{array}{l}\text { Raffination von Spei- } \\
\text { sefetten/-ölen; Her- } \\
\text { stellung hydrolysierter } \\
\text { Pflanzenproteine }\end{array}$ \\
\hline $\begin{array}{l}\text { Besonders betroffene } \\
\text { Lebensmittel }\end{array}$ & $\begin{array}{l}\text { Kartoffelchips, Pom- } \\
\text { mes frites, Brot, } \\
\text { Gebäck, Kaffee }\end{array}$ & $\begin{array}{l}\text { Kaffee, Brot, Räu- } \\
\text { cherwaren, Fleisch-, } \\
\text { Suppen-, Gemüse- } \\
\text { konserven, Babygläs- } \\
\text { chen }\end{array}$ & $\begin{array}{l}\text { Raffinierte Pflanzen- } \\
\text { fette/-öle, Margarine, } \\
\text { Säuglingsmilchnah- } \\
\text { rung }\end{array}$ & $\begin{array}{l}\text { Raffinierte Pflanzen- } \\
\text { fette/-öle, Margarine, } \\
\text { Säuglingsmilchnah- } \\
\text { rung }\end{array}$ & $\begin{array}{l}\text { Raffinierte Pflanzen- } \\
\text { fette/-öle, Margarine, } \\
\text { Säuglingsmilchnahrung }\end{array}$ \\
\hline $\begin{array}{l}\text { Sensitivster Endpunkt } \\
\text { (Tierexperiment) }\end{array}$ & $\begin{array}{l}\text { Tumoren in Harder- } \\
\text { schen Drüsen (Maus) }\end{array}$ & $\begin{array}{l}\text { Mesotheliome in } \\
\text { Nebenhoden und } \\
\text { Hoden (Ratte) }\end{array}$ & $\begin{array}{l}\text { Tumoren in Tunica va- } \\
\text { ginalis und Peritoneum } \\
\text { (Ratte) }\end{array}$ & $\begin{array}{l}\text { Hyperplasien in Nieren- } \\
\text { tubuli (Ratte) }\end{array}$ & Keine Daten \\
\hline $\begin{array}{l}\text { Potenzieller } \\
\text { Wirkmechanismus }\end{array}$ & $\begin{array}{l}\text { Gentoxisches Kan- } \\
\text { zerogen (toxischer } \\
\text { Metabolit Glycid- } \\
\text { amid) }\end{array}$ & $\begin{array}{l}\text { Mögliches gentoxi- } \\
\text { sches Kanzerogen } \\
\text { (toxischer Metabolit } \\
\text { cis-2-Buten-1,4-dial) }\end{array}$ & $\begin{array}{l}\text { Gentoxisches Kanzero- } \\
\text { gen (Glycidol selbst) }\end{array}$ & $\begin{array}{l}\text { Nicht bekannt (nicht } \\
\text { gentoxisch) }\end{array}$ & Nicht bekannt \\
\hline IARC-Einstufung & Gruppe $2 A^{a}$ & Gruppe $2 \mathrm{~B}^{\mathrm{b}}$ & Gruppe $2 A^{a}$ & Gruppe $2 B^{b}$ & - \\
\hline Regulierungsprinzip & $\begin{array}{l}\text { MoE-Konzept, } \\
\text { ALARA-Prinzip }\end{array}$ & - & $\begin{array}{l}\text { MoE-Konzept, } \\
\text { ALARA-Prinzip }\end{array}$ & Höchstwert & - \\
\hline
\end{tabular}

als gentoxisch, wobei N7-GlycidamidGuanin- und N3-Glycidamid-AdeninAddukte für die gentoxische Wirkung von Bedeutung sind. Gentoxische Effekte wurden auch in Nagetiermodellen bei Gaben hoher Acrylamidmengen beobachtet [9-11].

Im Tierversuch erwies sich die Langzeitapplikation von hohen Dosen Acrylamid als kanzerogen, wobei Tumore in einer Vielzahl von Geweben/ Organen, beispielsweise in den Brustdrüsen bei weiblichen Ratten und Mäusen, in der Schilddrüse bei der Ratte sowie in den Harderschen Drüsen (Tränendrüse) und der Lunge bei Mäusen auftraten. Dabei wurde als Referenzwert für die Wirkdosis ein $\mathrm{BMDL}_{10}$-Wert („benchmark dose lower confidence limit $10 \%$ “, siehe Infobox) von 0,17 mg/kg Körpergewicht und Tag für die Tumorentstehung in den Harderschen Drüsen von männlichen Mäusen als sensitivster Endpunkt berechnet [4]. Die International Agency for Research on Cancer (IARC) stufte Acrylamid in die Kategorie 2A als „Wahrscheinlich krebserregend für den Menschen" ein [12].

Die Aussagekraft der bisher durchgeführten epidemiologischen Studien bezüglich eines möglichen Zusammen- hangs zwischen Acrylamidexposition und der Entstehung von Krebs beim Menschen ist bisher nicht ausreichend für die Beurteilung dieser Frage $[4,5]$. Eine grundlegende Schwierigkeit besteht bei diesen Studien insbesondere in der Erfassung der langfristigen Acrylamidexposition. Auch eine erhöhte Krebsmortalität bei beruflich mit Acrylamid exponierten Arbeitern wurde bisher nicht beobachtet [4].

Eine aktuelle Expositionsabschätzung durch die Europäische Behörde für Lebensmittelsicherheit (EFSA) ergab für Erwachsene eine durchschnittliche Acrylamidbelastung von $0,5 \mu \mathrm{g} / \mathrm{kg}$ Körpergewicht und Tag und für Vielverzehrer (95. Perzentil) von $1,0 \mu \mathrm{g} / \mathrm{kg}$ Körpergewicht und Tag. Da Kinder bezogen auf ihr Körpergewicht einen höheren Energie- und Nährstoffbedarf haben, sind sie im jungen Alter um einen Faktor von etwa 2 höher exponiert als eine erwachsene Person [4].

Ein toxikologisch unbedenklicher Schwellenwert kann für Acrylamid aufgrund der gentoxischen und kanzerogenen Eigenschaften nicht abgeleitet werden. Für Verbindungen mit solchen Effekten kann zur Abschätzung des Gefährdungspotenzials das sog. Margin-of-
Exposure (MoE)-Konzept Anwendung finden (siehe Infobox). Für Acrylamid ergaben sich MoE-Werte von deutlich unter 1000 für alle Altersgruppen, wobei die Werte für Kinder und Kleinkinder niedriger sind als für Erwachsene [4]. Daher wird Acrylamid als gesundheitlich bedenkliche Substanz eingestuft, sodass es nach dem sog. As-low-as-reasonably-achievable (ALARA)-Prinzip bereits seit Jahren Bestrebungen gibt, die Acrylamidgehalte in Lebensmitteln $\mathrm{zu}$ minimieren.

In Deutschland wurde das Signalwertkonzept für bestimmte Lebensmittelgruppen mit dem Ziel eingeführt, die Acryamidgehalte in Lebensmitteln $\mathrm{zu}$ verringern. Dazu wurden in den jeweiligen Warengruppen die am höchsten belasteten Produkte identifiziert. Das 90. Perzentil wurde als Signalwert festgelegt. Wurde der Signalwert oder ein Gehalt von mehr als $1000 \mu \mathrm{g} / \mathrm{kg}$ (maximaler Signalwert) überschritten, so traten die Überwachungsbehörden der Länder in einen Minimierungsdialog mit den betroffenen Herstellern mit dem Ziel, den Acrylamidgehalt durch Änderungen der Rezeptur oder des Herstellungsverfahrens zu reduzieren. 
Im Jahr 2011 führte die Europäische Kommission Richtwerte („indicative values") ein, die auch in Deutschland die Signalwerte ablösen. Die Richtwerte sind in der Empfehlung der Kommission 2013/647/EU angegeben und betragen beispielsweise für verzehrfertige Pommes frites $600 \mu \mathrm{g}$ Acrylamid/kg Produkt und für Kartoffelchips aus frischen Kartoffeln und aus Kartoffelteig $1000 \mu \mathrm{g} / \mathrm{kg}$. Die EFSA stellte fest, dass eine zuverlässige europaweite Trendanalyse bezüglich einer Reduzierung des Acrylamidgehalts in Lebensmitteln aufgrund von Datenlücken und der nicht immer gegebenen Vergleichbarkeit von Ergebnissen aus verschiedenen Jahren nicht möglich ist. Dennoch zeigte ein Datensatz von Acrylamidmessungen aus 20 europäischen Ländern durchgeführt von Herstellern einen kontinuierlichen Abwärtstrend des Acrylamidgehalts in den Jahren 2002-2011 in Kartoffelchips [13]. Solch ein Trend war für andere Lebensmittelgruppen nicht erkennbar [4]. Zur Reduktion der individuellen Acrylamidexposition gilt weiterhin die Empfehlung, höher belastete Lebensmittel wie Pommes frites und Kartoffelchips nur in Maßen zu verzehren und auf eine schonende Zubereitung der Lebensmittel $\mathrm{zu}$ achten (Devise „Vergolden statt Verkohlen“). Zur Abschätzung der individuellen Acrylamidbelastung steht unter dem Link http://www.bfr.bund.de/de/ acrylamid__gesundheitliche_bewertung_ durch_das_bfr-1134.html ein Programm zur Bestimmung der täglichen mittleren Acrylamidaufnahme zur Verfügung (Acrylamidrechner).

\section{Monochlorpropandiol- und Glycidylfettsäureester}

$\mathrm{Zu}$ den Chlorpropandiolen zählen das 3-Monochlorpropandiol (3-MCPD) und das 2-Monochlorpropandiol (2-MCPD; - Abb. 1). Charakteristisch für diese beiden Substanzen ist, dass sie ein Glyceringrundgerüst aufweisen, bei dem eine Hydroxylgruppe in Position 3 bzw. 2 durch ein Chloratom ersetzt ist. Die Fettsäureester bestehen aus dem Chlorpropandiol, das mit einem oder zwei Fettsäureestern (Mono- und Diester) verestert ist. Bei Glycidol handelt es

Bundesgesundheitsbl 2017 -60:737-744 DOI 10.1007/s00103-017-2564-3

(c) Der/die Autor(en) 2017. Dieser Artikel ist eine Open-Access-Publikation.

\author{
S. Andres · K. Schultrich · B. Monien - T. Buhrke - N. Bakhiya · F. Frenzel · K. Abraham . \\ B. Schäfer · A. Lampen
}

\title{
Erhitzungsbedingte Kontaminanten in Lebensmitteln. Acrylamid, Furan und Fettsäureester von Monochlorpropandiolen und Glycidol
}

\section{Zusammenfassung}

Während der Herstellung und Zubereitung von Lebensmitteln können bei hohen

Temperaturen unerwünschte Verbindungen mit gesundheitsschädlichem Potenzial entstehen. Zu den am besten untersuchten erhitzungsbedingten Kontaminanten zählen Acrylamid, Furan sowie die Fettsäureester von Glycidol und Monochlorpropandiolen. Dieser Beitrag stellt die wesentlichen Erkenntnisse zur Entstehung, zur Toxikologie und zur Exposition dieser Verbindungen dar. Acrylamid und Glycidol haben sich in tierexperimentellen Untersuchungen als kanzerogen mit gentoxischem Mechanismus erwiesen; ihre Gehalte in Lebensmitteln sollten minimiert werden. Für 3-Monochlorpropandiol (3-MCPD) kann eine tolerierbare tägliche Aufnahmedosis abgeleitet werden. Dagegen ist für Furan und 2-MCPD aufgrund der ungenügenden Datenlage eine abschließende Risikobewertung derzeit noch nicht möglich.

Zusätzlich zu den genannten Verbindungen wurde eine Vielzahl weiterer erhitzungs- bedingter Substanzen in Lebensmitteln identifiziert, zu denen zumeist keine Daten zur Toxikologie und Exposition vorliegen. Daher kann für diese Verbindungen gegenwärtig keine Risikobewertung vorgenommen werden. Für eine Priorisierung dieser großen Anzahl an Verbindungen nach ihrem möglichen Gefährdungspotenzial erscheint zunächst eine Nutzung von Computermodellen sinnvoll, die anhand der chemischen Struktur einer Verbindung eine Vorhersage zu definierten toxikologischen Endpunkten erlaubt. Für Substanzen, die als prioritär eingestuft worden sind, sind jedoch für eine aussagekräftige Risikobewertung weitere, zumeist kosten- und zeitintensive Untersuchungen bezüglich der Toxikologie und der Quantifizierung dieser Verbindungen in Lebensmitteln notwendig.

Schlüsselwörter

Toxizität · Kanzerogene · Bewertungskonzepte $\cdot$ MCPD $\cdot$ Risikobewertung

\section{Heat-induced contaminants in foodstuffs. Acrylamide, furan, and fatty acid esters of monochloropropanediols and glycidol}

\section{Abstract}

The production and preparation of foodstuffs may entail at high temperatures the generation of undesirable, potentially harmful compounds. Among the best investigated heat-induced contaminants are acrylamide, furan, and the fatty acid esters of glycidol and the monochloropropanediols. This article presents the main insights into the formation, toxicology, and exposure of these compounds. Acrylamide and glycidol were characterized as carcinogens with a genotoxic mechanism in animal experiments. Their content in foods should be minimized. For 3-monochloropropanediol (3-MCPD), a tolerable daily intake can be derived. In contrast, a complete risk assessment is currently not possible for furan and 2-MCPD owing to insufficient data.

Many other heat-induced substances in foodstuffs were identified in addition to the compounds mentioned above, but for most no data on their toxicological properties and human exposure is available. Therefore, no risk assessment can currently be undertaken for these compounds. To prioritize this large number of compounds according to their possible hazard potential, it is reasonable to utilize computer modeling programs for the prediction of defined toxicological endpoints based on the molecular chemical structures. However, substances classed as a priority must be further investigated with regard to the toxicology and quantification of the food content of these compounds to allow a meaningful risk assessment.

Keywords

Toxicity · Carcinogens · Evaluation concepts . MCPD $\cdot$ Risk assessment 
<smiles>C=CC(N)=O</smiles>

Acrylamid<smiles>OCC(O)CCl</smiles>

3-MCPD<smiles>OCC(Cl)CO</smiles>

2-MCPD<smiles>c1ccoc1</smiles>

Furan

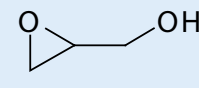

Glycidol
Abb. $1<$ Strukturformeln der erhitzungsbedingten Kontaminanten Acrylamid, Furan, 3-Monochlorpropandiol (MCPD), 2-MCPD und Glycidol sich um ein Glycerin, bei dem unter Ausbildung einer Epoxidstruktur ein Wassermolekül abgespalten wurde. Glycidylfettsäureester sind entsprechend Verbindungen aus Glycidol, das an der verbleibenden Hydroxylgruppe mit einer Fettsäure verestert ist.

MCPD- und Glycidylfettsäureester entstehen bei starker Erhitzung in fettund kochsalzhaltigen Lebensmitteln. Sie werden insbesondere bei der Raffination von pflanzlichen Fetten und Ölen während der sog. Desodorierung gebildet. Bei diesem Schritt werden bei hohen Temperaturen unerwünschte Geruchs- und Geschmacksstoffe entfernt. Die größte Expositionsquelle stellen somit raffinierte Fette und Öle bzw. die Lebensmittel dar, bei deren Herstellung diese Fette und Öle verwendet werden (z. B. Margarine oder Säuglingsmilchnahrung). Weitere Lebensmittel wie gebackene/geröstete Getreide- und Kartoffelprodukte enthalten ebenfalls MCPD-Fettsäureester [14-16]. Bereits 1978 waren unverestertes 3-MCPD und andere Chlorpropanole als relevante Kontaminanten in Sojasauce identifiziert worden. Durch technologische Maßnahmen wurde eine deutliche Senkung dieser Gehalte erreicht, sodass Sojasauce als Expositionsquelle gegenwärtig nicht mehr relevant ist [17].

\section{3-Monochlorpropandiol- fettsäureester}

Ungebundenes 3-MCPD erwies sich in tierexperimentellen Langzeitstudien als kanzerogen. 3-MCPD verursachte vermutlich über nicht gentoxische Wirkmechanismen insbesondere gutartige Nieren- und Hodentumore so- wie Fibroadenome der Brustdrüsen bei männlichen Ratten sowie ebenfalls Nierentumore bei weiblichen Ratten [16]. Die IARC stufte 3-MCPD als „,möglicherweise krebserregend für den Menschen“" ein (Gruppe 2B; [18]). In Anbetracht des kanzerogenen Gefährdungspotenzials von 3-MCPD war bis vor Kurzem nicht geklärt, inwieweit die mit Fettsäuren veresterten Verbindungen ein ähnliches Gefährdungspotenzial aufweisen wie freies 3-MCPD. Eine Kinetikstudie mit Ratten bestätigte die Erwartung, dass die Ester im Magen-Darm-Trakt gespalten werden und dabei fast vollständig ungebundenes 3-MCPD freisetzen [19, 20]. Eine subchronische Rattenstudie mit freiem 3-MCPD und einem 3-MCPDFettsäureester wies entsprechend auf ein nur leicht abgeschwächtes Gefährdungspotenzial des Esters an Niere und Hoden als den primären Zielorganen hin [21]. Daher erscheint es für die Risikobewertung gerechtfertigt, die Exposition gegenüber 3-MCPD-Fettsäureestern als äquimolare Exposition gegenüber 3-MCPD zu betrachten.

Das Joint FAO/WHO Expert Committee on Food Additives (JECFA) hatte 2002 aus der damals verfügbaren 3-MCPD-Langzeitstudie an Ratten einen Wert für die tolerierbare tägliche Aufnahme (TDI) von $2 \mu \mathrm{g} / \mathrm{kg}$ Körpergewicht abgeleitet. Das Bundesinstitut für Risikobewertung $(\mathrm{BfR})$ hatte diesen Wert 2012 unter Einbeziehung neuerer Daten bestätigt [22]. Hiervon abweichende TDI-Werte wurden 2016 von EFSA [16] und JECFA [23] veröffentlicht. Die 3 zuletzt genannten Ableitungen wurden auf Basis der gleichen Langzeitstudie an Ratten vorgenommen, mit der Bildung von tubulären Hyperplasien als sensitivsten Endpunkt. Die Differenzen in den abgeleiteten TDI-Werten resultieren primär aus der Verwendung unterschiedlicher Modelle bei der mathematischen Modellierung der Dosis-Wirkung-Beziehung (Benchmark-Modellierung, siehe Infobox). Möglicherweise ergibt sich eine Klärung durch die kürzlich von EFSA gegebene Empfehlung, nicht einzelne Modelle, sondern ein Modell-Averaging $\mathrm{zu}$ verwenden [24].

Aktuelle Expositionsabschätzungen der EFSA ergeben für 3-MCPD bei erwachsenen Personen einen mittleren Wert von $0,3 \mu \mathrm{g} / \mathrm{kg}$ Körpergewicht und Tag und für Vielverzehrer (95. Perzentil) von $0,7 \mu \mathrm{g} / \mathrm{kg}$ Körpergewicht und Tag. Im Vergleich zu Erwachsenen weisen Kleinkinder und Kinder (3-10 Jahre) eine etwa 2-fach höhere Exposition auf. Nicht gestillte Säuglinge, die ausschließlich über Säuglingsmilchnahrung ernährt werden, sind unter Berücksichtigung von mittleren Gehaltsdaten an 3-MCPD mit im Schnitt etwa 2,4 $\mu \mathrm{g} / \mathrm{kg}$ Körpergewicht und Tag deutlich höher gegenüber 3-MCPD exponiert als Erwachsene. Bei Berücksichtigung des 95. Perzentils der Gehaltsdaten an 3-MCPD sowie unter der Annahme von Markentreue wurde für 3-MCPD eine Exposition für nicht gestillte Säuglinge von $3,2 \mu \mathrm{g} / \mathrm{kg}$ Körpergewicht und Tag ermittelt [16].

In den letzten Jahren wurde bereits eine Vielzahl an Möglichkeiten zur Minimierung von 3-MCPD-Fettsäureestern in Lebensmitteln veröffentlicht (z. B. durch die Auswahl geeigneter Rohstoffe, Anpassung von Raffinationsbedingungen oder nachträgliche Reduktion der Prozesskontaminanten; [25]). Dennoch wurden hinsichtlich des Gehalts insbesondere von 3-MCPD-Fettsäureestern in Palmöl und Palmfett zwischen 2010 und 2015 nur minimale Fortschritte erzielt [16]. Die individuelle Exposition gegenüber MCPD-Fettsäureestern kann vermindert werden, indem raffinierte Fette und Margarine nur in Maßen verzehrt werden. Da für nicht gestillte Säuglinge keine geeigneten Alternativen bei der Säuglingsernährung verfügbar sind, wird Müttern, die nicht stillen können, geraten, ihre Säuglinge weiterhin 


\section{Infobox \\ Margin-of-Exposure-Konzept und Benchmark-Modellierung}

Das Margin-of-Exposure(MoE)-Konzept ist zur Abschätzung des potenziellen Risikos von gentoxisch und kanzerogen wirkenden Substanzen international etabliert und wird in einem von der Europäischen Behörde für Lebensmittelsicherheit (EFSA) erarbeiteten Gutachten als harmonisierter Ansatz für die Risikobewertung von Substanzen in Lebensmitteln mit gentoxischen und kanzerogenen Eigenschaften empfohlen. Der MoE-Wert ist eine dimensionslose Zahl, die das Verhältnis einer Wirkdosis im Tierversuch, bei der eine geringfügige, aber messbare nachteilige Wirkung beobachtet wird, und Schätzungen zur Höhe der Exposition gegenüber der betreffenden Substanz unter Berücksichtigung unterschiedlicher Verzehrmuster beschreibt. Mit diesem Konzept wird versucht, das Ausmaß möglicher Risiken verschiedener in Lebensmitteln vorkommender gentoxischer Kanzerogene vergleichend darzustellen. Dies gilt unter der Annahme, dass der Kurvenverlauf der Beziehung zwischen krebsauslösender Wirkung und Dosis bei den verschiedenen Substanzen vergleichbar ist.

Nach diesem von der EFSA vorgeschlagenen Konzept können für die Referenzwerte der Wirkdosis aus dem Tierexperiment 2 Parameter berechnet werden. Zum einen die Benchmarkdosis $B M_{10}$ („,benchmark dose lower confidence limit $10 \%$ "), bei der eine gegenüber Kontrolltieren $10 \%$ höhere Inzidenz von Krebs in einem bestimmten Gewebe auftritt. Die Berechnung setzt die Annahme eines konkreten mathematischen Modells für die Dosis-Wirkung-Beziehung voraus und ist nur sinnvoll bzw. möglich, wenn genügend Daten mit verschiedenen Dosierungen zur Verfügung stehen. Damit die Besorgnis bezüglich eines möglichen Krebsrisikos für den Menschen als gering eingestuft werden kann, sollte ein auf $\mathrm{BMDL}_{10}$ bezogener MoE-Wert größer als 10.000 sein. Als weiterer Referenzwert für die Wirkdosis kann auch ein T25-Wert bestimmt werden, der die Dosis angibt, die innerhalb der Lebensspanne bei $25 \%$ der Tiere in einem bestimmten Gewebe Krebs verursacht. Bei der Berechnung muss eine Korrektur bezüglich der spontan auftretenden Krebsrate in diesem Gewebe erfolgen. Damit die Besorgnis bezüglich eines möglichen Krebsrisikos für den Menschen als gering eingestuft werden kann, sollte ein auf T25 bezogener MoE-Wert größer als 25.000 sein. Je niedriger der MoE-Wert einer bestimmten Substanz unterhalb der oben genannten Werte liegt, desto größer sind die gesundheitlichen Bedenken bezüglich eines Krebsrisikos für den Menschen [16, 40].

mit den herkömmlichen Produkten zu füttern.

\section{2-Monochlorpropandiolfettsäu- reester}

Das Gefährdungspotenzial von 2-MCPD lässt sich aufgrund von sehr begrenzt vorliegenden toxikologischen Daten derzeit nicht abschätzen. Es fehlen insbesondere Daten aus chronischen Toxizitätsstudien. Die wenigen verfügbaren Informationen zur Toxizität von 2-MCPD weisen auf ein Toxizitätsprofil hin, das sich von dem des 3-MCPD unterscheidet. Möglicherweise sind Unterschiede im Metabolismus dieser beiden Substanzen hierfür verantwortlich. Im Gegensatz zu 2-MCPD werden in vivo aus 3-MCPD die Metabolite $\beta$-Chlorlactaldehyd und $\beta$-Chlorlactat gebildet, die möglicherweise eine Rolle bei der Ausprägung der Nierentoxizität nach 3-MCPD-Gabe spielen [15, 16]. Der Gehalt von 2-MCPD in Lebensmitteln ist um etwa die Hälfte bis ein Drittel niedriger als der 3-MCPD-Gehalt, sodass sich für 2-MCPD niedrigere Expositionen im Mittel von etwa 0,1 $\mu \mathrm{g} / \mathrm{kg}$ Körpergewicht und Tag und bei hohem Verzehr von
$0,3 \mu \mathrm{g} / \mathrm{kg}$ Körpergewicht und Tag ergeben [16].

\section{Glycidylfettsäureester}

Analog zu den MCPD-Fettsäureestern entstehen die Fettsäureester des strukturell verwandten Glycidols (• Abb. 1) insbesondere bei der Raffination von pflanzlichen Fetten und Ölen während der Desodorierung. Daher finden sich Glycidylfettsäureester wie die MCPDFettsäureester vor allem in raffinierten Ölen und Fetten sowie den entsprechenden Lebensmitteln, bei deren Herstellung diese Fette verwendet werden $[14,16]$. Auch die Glycidylfettsäureester werden durch Esterasen im Magen-Darm-Trakt fast vollständig hydrolysiert, sodass bei der Risikobewertung der Fettsäureester von einem ähnlichen Gefährdungspotenzial ausgegangen werden kann wie bei freiem Glycidol [26].

Im Gegensatz zu 3-MCPD erwies sich das Epoxid Glycidol als gentoxisch. Die Langzeitapplikation hoher Dosierungen von Glycidol führte zur Tumorentstehung in verschiedenen Organen/Geweben wie Hoden, Brustdrüse,
Gehirn und Schilddrüse bei der Ratte $[14,16]$. Das BfR und die EFSA bestimmten als Referenzpunkt für die Wirkdosis einen T25-Wert (siehe Infobox) für peritoneale Mesotheliome der Hoden bei männlichen Ratten von 10,2 mg/kg Körpergewicht und Tag $[16,27]$. Die IARC klassifizierte Glycidol als „wahrscheinlich krebserregend für den Menschen" (Gruppe 2A; [28]).

Für die Exposition von Glycidol ergibt sich im Mittel für Erwachsene ein Wert von $0,2 \mu \mathrm{g} / \mathrm{kg}$ Körpergewicht und Tag und für Vielverzehrer von $0,5 \mu \mathrm{g} / \mathrm{kg}$ Körpergewicht und Tag. Im Vergleich zum Erwachsenen weisen Kleinkinder und Kinder (3-10 Jahre) eine etwa 2-fach höhere Exposition für diese Verbindung auf. Für nicht gestillte Säuglinge, die ausschließlich über Säuglingsmilchnahrung ernährt wurden, wurde unter Berücksichtigung von mittleren Gehaltsdaten an Glycidol eine Exposition von $1,9 \mu \mathrm{g} / \mathrm{kg}$ Körpergewicht und Tag ermittelt. Bei Berücksichtigung des 95 . Perzentils der Gehaltsdaten sowie unter der Annahme von Markentreue wurde für Glycidol eine Exposition für nicht gestillte Säuglinge von $4,9 \mu \mathrm{g} / \mathrm{kg}$ Körpergewicht und Tag ermittelt [16].

Für Glycidol kann aufgrund der gentoxischen und kanzerogenen Eigenschaften kein toxikologisch unbedenklicher Schwellenwert abgeleitet werden. Ähnlich wie beim Acrylamid findet daher das MoE-Konzept Anwendung (siehe Infobox), wobei für Glycidol die MoE-Werte durch die Anwendung des T25-Werts als Wirkdosis über 25.000 liegen sollten, damit die Besorgnis bezüglich eines möglichen Krebsrisikos für den Menschen als gering eingestuft werden kann. Für Glycidol ergaben sich MoE-Werte von unter 25.000 vor allem bezüglich einer mittleren und hohen Exposition für Kleinkinder und Kinder (3-10 Jahre) sowie bei hohem Verzehr für Jugendliche und Erwachsene. Für nicht gestillte Säuglinge, die ausschließlich über Säuglingsmilchnahrung ernährt wurden, wurden die niedrigsten MoE-Werte von 5400 bzw. 2100 unter Berücksichtigung des mittleren bzw. hohen Glycidol-Gehalts ermittelt, sodass gesundheitliche Bedenken bezüglich eines Krebsrisikos für den Menschen bestehen [16]. 
Wie für Acrylamid gilt für Glycidylfettsäureester das ALARA-Prinzip, wobei sich das Minimierungskonzept insbesondere auf Raffinationsprozesse bezieht. Die bereits bei den 3-MCPD-Fettsäureestern beschriebenen Methoden zur Minimierung finden ebenfalls bei der Reduzierung des Gehalts der Glycidylfettsäureester Anwendung [25], wodurch deren Gehalt in Palmöl und Palmfett zwischen 2010 und 2015 um mehr als die Hälfte reduziert wurde [16]. Die für 3-MCPDFettsäureester gemachten Verzehrempfehlungen gelten analog für Glycidylfettsäureester.

\section{Furan}

Furan (•Abb. 1) ist eine heterozyklische, aromatische, leicht flüchtige Verbindung, die im Lebensmittel beim Erhitzen aus Kohlenhydraten, Aminosäuren, Ascorbinsäure und ungesättigten Fettsäuren entsteht [29]. Es wird insbesondere beim Rösten von Lebensmitteln oder bei Erhitzungsvorgängen in geschlossenen $\mathrm{Ge}$ fäßen gebildet. Belastete Lebensmittelgruppen sind im Wesentlichen gerösteter Kaffee, Instantkaffee, Babygläschen, Suppen, Saucen sowie Fleisch- und Getreideprodukte [30, 31].

Furan wird im Magen-Darm-Trakt schnell resorbiert und insbesondere durch CYP2E1 in der Leber zu dem toxischen Metaboliten (2Z)-2-Butendial (cis-2-Buten-1,4-dial) verstoffwechselt. Dieser Dialdehyd reagiert mit Thiolen (z. B. Glutathion) und mit Aminogruppen von Proteinen. Zwar wurden DNAAddukte mit dem Dialdehyd in vitro beobachtet; jedoch in vivo in furanbehandelten Ratten bisher nicht nachgewiesen [32]. Studien zur Gentoxizität von Furan lieferten keine eindeutigen Ergebnisse. Zwar erwies sich Furan selbst auch in Anwesenheit eines Systems zur externen Metabolisierung als nicht mutagen in In-vitro-Testsystemen [33], jedoch wurde sein reaktiver Aldehyd cis-2-Buten1,4-dial positiv auf Mutagenität getestet $[34,35]$. Die Kanzerogenität von Furan wurde in mehreren Langzeittierstudien untersucht. Die orale Gabe von Furan über 2 Jahre erhöhte die Inzidenzen unterschiedlicher Neoplasien in Versuchstieren. Deutlich war die Zunahme der Inzidenz von hepatozellulären Adenomen und Karzinomen bei Mäusen und Ratten beiderlei Geschlechts [33, 36]. In einer jüngeren Studie wurden außerdem Mesotheliome in Nebenhoden und Hoden bei Ratten beobachtet $[33,36]$. Es ist derzeit jedoch unklar, welche Mechanismen die Kanzerogenität von Furan bedingen, wodurch die Abschätzung der Relevanz dieser Tierversuchsdaten für ein mögliches kanzerogenes Risiko beim Menschen erschwert wird. Die IARC stufte Furan als „möglicherweise krebserregend für den Menschen“ ein (Gruppe 2B; [37]).

Auf Basis der Gehaltsangaben für die oben genannten Lebensmittelgruppen sowie Expositionsdaten aus verschiedenen Ernährungsstudien schätzte die EFSA die durchschnittliche Exposition für Erwachsene, berechnet für verschiedene Länder, auf 0,03-0,59 $\mu$ g Furan/kg Körpergewicht und für Vielverzehrer (95. Perzentil) auf 0,09-1,29 $\mu \mathrm{g} / \mathrm{kg}$ Körpergewicht und Tag, wobei Kaffee die hauptsächliche Expositionsquelle von bis zu fast $90 \%$ der Gesamtexposition darstellt. Für Kleinkinder beträgt die mittlere Exposition 0,05-0,31 $\mu \mathrm{g}$ Furan/kg Körpergewicht und Tag und bei hoher Exposition (95. Perzentil) 0,2-1,38 $\mu \mathrm{g} / \mathrm{kg}$ Körpergewicht und Tag. Die EFSA verwies dabei auf die unzureichende Datenlage zu Furangehalten in wärmebehandelten Lebensmitteln. Es fehlen insbesondere analytische Daten zu den Gehalten vor und nach der Zubereitung von Nahrungsmitteln bei hohen Temperaturen [6]. Für eine abschließende Risikobewertung von Furan sind diese Daten sowie die oben erwähnten toxikologischen Daten zum Wirkmechanismus unabdingbar.

Auch wenn derzeit das gesundheitliche Gefährdungspotenzial von Furan nicht abschließend geklärt ist, könnte individuell die Furanexposition minimiert werden, indem vorwiegend frisch zubereitete Lebensmittel inklusive frisch zubereiteter Babynahrung und weniger Fertiggerichte und Konserven verzehrt werden. Da Furan eine leicht flüchtige Verbindung darstellt, wird weiterhin empfohlen, Fertiggerichte und Babygläschen im offenen Gefäß zu erwärmen und regelmäßig umzurühren. Darüber hinaus bleibt zu beachten, dass für Erwachsene der Kaffeekonsum die höchste Expositionsquelle für Furan darstellt.

\section{Toxikologisch wenig charak- terisierte erhitzungsbedingte Kontaminanten}

Die unüberschaubar große Anzahl von Substanzen, die beim Erhitzen von Lebensmitteln entstehen können, stellt eine besondere Herausforderung bei der Bewertung erhitzungsbedingter Kontaminanten dar. So umfasst allein die Gruppe der aus der Maillard-Reaktion und der Lipidoxidation resultierenden erhitzungsbedingten Reaktionsprodukte mehrere hundert Verbindungen. Eine Risikobewertung aller Verbindungen ist jedoch nicht möglich, da von den meisten dieser Verbindungen derzeit nicht viel mehr als ihre chemische Struktur bekannt ist. Für eine aussagekräftige Risikobewertung ist allerdings ein umfassender Datensatz zur Toxizität und zu den Gehalten dieser Verbindungen in Lebensmitteln erforderlich. Die hier ausgeführten Beispiele (Acrylamid, MCPDund Glycidylfettsäureester und Furan) haben in der jüngeren Vergangenheit gezeigt, dass es nach der Entdeckung einer bisher unbekannten Substanz in Lebensmitteln mehrere Jahre dauert, um die für eine Risikobewertung notwendigen Daten zu erheben. Es kann dann noch weitere Jahre dauern, bis geeignete Risikomanagementmaßnahmen greifen und die Gehalte in den Lebensmitteln tatsächlich abgesenkt werden können, da die entsprechenden technologischen Möglichkeiten zunächst identifiziert werden müssen.

Angesichts der großen Anzahl von Substanzen, die bisher noch nicht bewertet werden konnten, könnte in einem ersten Schritt zunächst eine Priorisierung der Stoffe nach ihrem möglichen Gefährdungspotenzial angestrebt werden. Ein interessanter Ansatz ist dabei die Nutzung von Computermodellen, die anhand der chemischen Struktur einer Verbindung eine Vorhersage zu deren Toxizität im Hinblick auf verschiedene relevante Endpunkte erlauben. Innerhalb der letzten Jahrzehnte sind verschiedene Computermodelle entwickelt worden, 
um In-silico-Vorhersagen für diverse Chemikaliencharakteristika zu ermöglichen. Alle diese Modelle basieren auf der Annahme, dass sich die physikochemischen und sogar die toxischen Eigenschaften von chemischen Verbindungen aus deren Molekülstruktur ableiten lassen. Für einen sog. Read-across-Ansatz werden spezielle Molekülstrukturen betrachtet, um bestimmte, nicht bekannte biologische Aktivitäten oder stoffliche Eigenschaften von wenig charakterisierten Verbindungen anhand der bekannten Eigenschaften von Verbindungen mit vergleichbarer Struktur vorherzusagen. Die Substanzen können dabei auf Basis struktureller Ähnlichkeiten gruppiert werden, die entweder gemeinsame funktionelle Gruppen, gemeinsame Ausgangsstoffe und/oder ähnliche Abbauprodukte oder ein bestimmtes festes Wirkmuster aufweisen.

Darüber hinaus wurden auch komplexe mathematische Methoden zur Vorhersage der „(quantitative) structure activity relationship" ([Q]SAR) entwickelt [38]. Diese Computermodelle basieren auf den bereits experimentell erhobenen Daten gut untersuchter Substanzen. Was bereits vor etwa 50 Jahren mit einfachen Regressionsmodellen mit einem Vergleich von wenigen gleichartigen Verbindungen angefangen hat [39], entwickelte sich bis heute zu statistischen, komplexen Methoden basierend auf umfangreichen Datensätzen mit mehreren 1000 unterschiedlichen Molekülen. Trotzdem kann es aufgrund der Tatsache, dass sich die zugrunde liegenden mathematischen Algorithmen und die verschiedenen Trainingssets, mit denen die Modelle entwickelt worden sind, dazu kommen, dass es je nach angewandtem Modell zu einer unterschiedlichen Vorhersage bezüglich eines definierten Endpunkts kommt. Zur Verfügung stehende Endpunkte sind dabei beispielweise biologische Aktivitäten wie Mutagenität, Kanzerogenität, Hautsensibilisierung oder Entwicklungstoxizität oder physikochemische Eigenschaften wie Dichte und Siedetemperatur. (Q)SAR-Modelle finden bereits breite Anwendung in Wissenschaft, Industrie und behördlichen Einrichtungen.

Hinsichtlich der Vorhersage toxischer Eigenschaften existieren die derzeit bes- ten Computermodelle für vor allem vergleichsweise einfache Endpunkte wie z. B. der Mutagenität. Dieser Endpunkt bildet die Fähigkeit einer Substanz ab, mit der DNA chemisch zu reagieren, sodass hier letztendlich die chemische Reaktivität durch das Modell bewertet werden muss. Hier sei jedoch angemerkt, dass nur die Verbindung an sich mithilfe der In-silico-Methoden analysiert wird. Potenziell toxische Metabolite, die in vivo gebildet werden, bleiben jedoch unberücksichtigt. Die Computermodelle zur Mutagenität haben auch nur eine Sensitivität und Spezifität von etwa $90 \%$, etwa $10 \%$ aller Vorhersagen sind somit falsch. Noch deutlich schlechter ist die Vorhersagekraft von Computermodellen zu komplexen Endpunkten wie z. B. der Kanzerogenität. Die Krebsentstehung ist ein sehr komplexer multifaktorieller Prozess. Chemische Verbindungen könnten vielfältig auf diesen Prozess der Krebsentstehung einwirken (z. B. auf die Tumorinitiierung, -promotion oder -progression). Weitere Faktoren wie beispielsweise Speziesunterschiede können die Vorhersagekraft bezüglich der Kanzerogenität einer bestimmten Substanz weiter erschweren. Nichtsdestotrotz gibt es erste Ansätze, die Vorhersagekraft durch die Kombination verschiedener Modelle zu verbessern [3]. So kann die kombinierte Nutzung derartiger Computermodelle kurzfristig eine erste Abschätzung des toxischen Potenzials einer Substanz erlauben.

Diejenigen Substanzen, die beispielsweise mithilfe der Computermodelle als gentoxisch und kanzerogen eingestuft worden sind, können hiermit als höchst prioritäre Verbindungen für weiterführende Untersuchungen angesehen werden. Letztendlich dienen die Insilico-Verfahren jedoch lediglich einer Priorisierung der Vielzahl an identifizierten Kontaminanten in Lebensmitteln. Für eine umfassende aussagekräftige Risikobewertung sind eine vollständige Charakterisierung der Toxizität der jeweiligen Substanz sowie die Abschätzung der Exposition notwendig. Mithilfe von In-vitro- und tierexperimentellen Untersuchungen sind Aussagen zur Toxikokinetik, Mutagenität, Kanzerogenität und zur Organ-, Reproduktions- und
Entwicklungstoxizität sowie zu möglichen Wirkmechanismen $\mathrm{zu}$ treffen. Zur Bestimmung der Exposition sind die Gehaltdaten vor allem in hoch belasteten Lebensmitteln erforderlich. Insbesondere die tierexperimentellen Untersuchungen sowie die Etablierung von chemisch-analytischen Verfahren zur Quantifizierung der Substanzen und deren regelmäßige Gehaltmessungen in den verschiedenen Lebensmitteln sind sehr kostenintensive und zeitaufwendige Verfahren. Dennoch können erst auf Basis einer umfassenden aussagekräftigen Risikobewertung Maßnahmen für das Risikomanagement festgelegt werden, um einen bestmöglichen Verbraucherschutz zu gewährleisten.

\section{Korrespondenzadresse}

PD Dr. K. Abraham

Bundesinstitut für Risikobewertung

Max-Dohrn-Str. 8-10, 10589 Berlin, Deutschland

klaus.abraham@bfr.bund.de

\section{Einhaltung ethischer Richtlinien}

Interessenkonflikt. S. Andres, K. Schultrich, B. Monien, T. Buhrke, Nadiya Bakhiya, F. Frenzel, K. Abraham, B. Schäfer und A. Lampen geben an, dass kein Interessenkonflikt besteht.

Dieser Beitrag beinhaltet keine von den Autoren durchgeführten Studien an Menschen oder Tieren.

Open Access. Dieser Artikel wird unter der Creative Commons Namensnennung 4.0 International Lizenz (http://creativecommons.org/licenses/by/4.0/deed. de) veröffentlicht, welche die Nutzung, Vervielfältigung, Bearbeitung, Verbreitung und Wiedergabe in jeglichem Medium und Format erlaubt, sofern Sie den/die ursprünglichen Autor(en) und die Quelle ordnungsgemäß nennen, einen Link zur Creative Commons Lizenz beifügen und angeben, ob Änderungen vorgenommen wurden.

\section{Literatur}

1. van Boekel M, Fogliano V, Pellegrini N, Stanton C, Scholz G, Lalljie S et al (2010) A review on the beneficial aspects of food processing. Mol Nutr Food Res 54:1215-1247

2. Appel KE, Abraham K (2010) Chemische Lebensmittelsicherheit Nationale und europäische Aspekte. Bundesgesundheitsblatt Gesundheitsforschung Gesundheitsschutz 53:534-542

3. Frenzel F, Buhrke T, Wenzel I, Andrack J, Hielscher J, Lampen A (2017) Use of in silico models for prioritization of heat-induced food contaminats in mutagenicity and carcinogenicity testing. Arch Toxicol. doi:10.1007/s00204-016-1924-3 
4. European Food Safety Authority (2015) Scientific Opinion on acrylamide in food, EFSA Panel on Contaminants in the Food Chain (CONTAM). EFSA J 13:4104

5. BfR (Bundesinstitut für Risikobewertung) (2013) Acrylamid in Lebensmitteln. Stellungnahme Nr. 043/2011 des BfR vom 29. Juni 2011, ergänzt am 21. Januar 2013

6. EFSA (European Food Safety Authority) (2011) Results on acrylamide levels in food from monitoring years 2007-2009 and exposure assessment. EFSA J 9:2133

7. Duale N, Bjellaas T, Alexander J, Becher G, Haugen M, Paulsen JE et al (2009) Biomarkers of human exposure to acrylamide and relation to polymorphisms in metabolizing genes. Toxicol Sci 108:90-99

8. Sweeney LM, Kirman CR, Gargas ML, Carson ML, TardiffRG (2010) Development of a physiologicallybased toxicokinetic model of acrylamide and glycidamide in rats and humans. Food Chem Toxicol 48:668-685

9. Ghanayem BI, Witt KL, Kissling GE, Tice RR, Recio $L$ (2005) Absence of acrylamide-induced genotoxicity in CYP2E1-null mice: evidence consistent with a glycidamide-mediated effect. Mutat Res 578:284-297

10. Manjanatha MG, Aidoo A, Shelton SD, Bishop ME, McDaniel LP, Lyn-Cook LE et al (2006) Genotoxicity of acrylamide and its metabolite glycidamide administered in drinking water to male and female Big Blue mice. Environ Mol Mutagen 47:6-17

11. Sanchez J, Cabrer JM, Rossello CA, Palou A, Pico $C$ (2008) Formation of hemoglobin adducts of acrylamide after its ingestion in rats is dependent on age and sex. J Agric Food Chem 56:5096-5101

12. IARC (International Agency for Research on Cancer) (1994) IARC monographs on the evaluation of the carcinogenic risk of chemicals to humans: Acrylamide Bd.60., S389-433

13. Powers SJ, Mottram DS, Curtis A, Halford NG (2013) Acrylamide concentrations in potato crisps in Europe from 2002 to 2011. Food Addit Contam Part A Chem Anal Control Expo Risk Assess 30:1493-1500

14. Bakhiya N, Abraham K, Gürtler R, Appel KE, Lampen A (2011) Toxicological assessment of 3-chloropropane-1,2-diol and glycidol fatty acid esters in food. Mol Nutr Food Res 55:509-521

15. Andres S, Appel KE, Lampen A (2013) Toxicology, occurrence and risk characterisation of the chloropropanols in food: 2-Monochloro-1,3-propanediol, 1,3-dichloro-2-propanol and 2,3-dichloro-1propanol. Food Chem Toxicol 58C:467-478

16. European Food Safety Authority (2016) Risks for human health related to the presence of 3-and 2-monochloropropanediol (MCPD), and their fatty acid esters, and glycidyl fatty acid esters in food, EFSA Panel on Contaminants in the Food Chain (CONTAM). EFSA J 14:4426

17. Lee BQ, Khor SM (2015) 3-Chloropropane-1,2diol (3-MCPD) in soy sauce: a review on the formation, reduction, and detection of this potential carcinogen. Compr Rev Food Sci Food Saf 14:48-66

18. IARC (International Agency for Research on Cancer) (2013) IARC monographs on the evaluation of the carcinogenic risk of chemicals to humans: 3-Monochloro-1,2-propanediol Bd. 101. S349-374

19. Abraham K, Appel KE, Berger-Preiss E, Apel E, Gerling S, Mielke H et al (2013) Relative oral bioavailability of 3-MCPD from 3-MCPD fatty acid esters in rats. Arch Toxicol 87:649-659
20. BuhrkeT,WeisshaarR,Lampen A(2011)Absorption and metabolism of the food contaminant 3-chloro1,2-propanediol (3-MCPD) and its fatty acid esters by human intestinal Caco- 2 cells. Arch Toxicol 85:1201-1208

21. Barocelli E, Corradi A, Mutti A, Petronini PG (2011) Comparison between 3-MCPD and its palmitic esters in a 90-day toxicological study. EFSA Support Publ. doi:10.2903/sp.efsa.2011.en-187

22. BfR (Bundesinstitut für Risikobewertung) (2012) 3-MCPD-Fettsäureester in Lebensmitteln. Stellungnahme $\mathrm{Nr}$ 006/2013 des BfR vom 3 April 2012

23. JECFA (Joint FAO/WHO Expert Committee on Food Additives) (2016) Joint FAO/WHO expert committee on food additives, Eighty-third meeting, Rome, 8-17 November 2016, Summary and conclusions, issued 23 November 2016.

24. European Food Safety Authority (2017) Update: use of the benchmark dose approach in risk assessment. EFSA J 15:4658

25. Matthäus B, Pudel F (2014) Mitigation of MCPD and glycidyl esters in edible oils. Processing contaminants in edible oils: MCPD and glycidyl esters. Elsevier AOCS Press, Illinois, S23-55

26. Appel KE, Abraham K, Berger-Preiss E, Hansen T, Apel E, Schuchardt S et al (2013) Relative oral bioavailability of glycidol from glycidyl fatty acid esters in rats. Arch Toxicol 87:1649-1659

27. BfR (Bundesinstitut für Risikobewertung) (2009) Erste Einschätzung zur Bewertung der in raffinierten pflanzlichen Fetten nachgewiesenen Gehalte von Glycidol-Fettsäureestern. Stellungnahme Nr. 007/2009 des BfR vom 10. März 2009

28. IARC (International Agency for Research on Cancer) (2000) IARC monographs on the evaluation of the carcinogenic risk of chemicals to humans: Glycidol Bd. 77., S469-486

29. Crews C, Castle L (2007) A review of the occurrence, formation and analysis of furan in heat-processed foods. Trends Food Sci Technol 18:365-372

30. Bakhiya N, Appel KE (2010) Toxicity and carcinogenicity of furan in human diet. Arch Toxicol 84:563-578

31. European Food Safety Authority (2011) Update on furan levels in food from monitoring years 2004-2010 and exposure assessment. EFSA J 9:2347-2379

32. Churchwell MI, Scheri RC, Von Tungeln LS, Gamboa da Costa G, BelandFA, Doerge DR (2015) Evaluation of serum and liver toxicokinetics for furan and liver DNA adduct formation in male Fischer 344 rats. Food Chem Toxicol 86:1-8

33. NTP (U.S. National Toxicology Program) (1993) Toxicology and carcinogenesis studies of furan (CAS no. 110-00-9) in F344/N rats and ${\mathrm{B} 6 C 3 \mathrm{~F}_{1} \text { mice }}^{-1}$ (Gavage studies). Techn Rep Ser No. 402

34. Peterson LA, Naruko KC, Predecki DP (2000) A reactive metabolite of furan, cis-2-butene-1,4-dial, is mutagenic in the Ames assay. Chem Res Toxicol 13:531-534

35. Kellert M, Brink A, Richter I, Schlatter J, Lutz WK (2008) Tests for genotoxicity and mutagenicity of furan and its metabolite cis-2-butene-1,4-dial in $\mathrm{L} 5178 \mathrm{Y} \mathrm{tk}^{+/-}$mouse lymphoma cells. Mutat Res 657:127-132

36. Von Tungeln LS, Walker NJ, Olson GR, Mendoza MC, Felton RP, Thorn BT et al (2017) Low dose assessment of the carcinogenicity of furan in male F344/N Nctr rats in a 2-year gavage study. Food Chem Toxicol 99:170-181

37. IARC (International Agency for Research on Cancer) (1995) IARC monographs on the evaluation of the carcinogenic risk of chemicals to humans: Furan Bd. 63., S393-407

38. Cronin MTD (2010) Quantitative Structure Activity Relationships (QSARs): applications and methodology. In: Puzyn T, Leszczynski J, Cronin MT (Hrsg) Recent advances in QSAR studies: methods and applications. Springer, Dordrecht, S3-11

39. Hansch C, Maloney PP, Fujita T, Muir RM (1962) Correlation of biological activity of phenoxyacetic acids with Hammett substituent constants and partition coefficients. Nature 194:178-180

40. European Food Safety Authority (2005) Opinion of the Scientific Committee on a request from EFSA related to a harmonised approach for risk assessment of substances which are both genotoxic and carcinogenic. EFSA J 282:1-31 